\title{
Receptance-based partial pole assignment for asymmetric systems using state-feedback
}

\author{
M. Ghandchi Tehrani ${ }^{\mathrm{a}}$ and H. Ouyang ${ }^{\mathrm{b}, *}$ \\ ${ }^{\mathrm{a}}$ Institute of Sound and Vibration Research, University of Southampton, Highfield, Southampton, UK \\ ${ }^{\mathrm{b}}$ School of Engineering, University of Liverpool, The Quadrangle, Liverpool, UK
}

\begin{abstract}
Many structures or machines interact with some internal nonconservative forces and present asymmetric systems in which the stiffness and damping matrices are asymmetric. Examples include friction-induced vibration and aeroelastic flutter. Asymmetric systems are prone to flutter instability as a result of the real parts of some poles becoming positive when certain system parameters vary.

This paper presents a receptance-based inverse method for assigning a number of complex poles of second-order damped asymmetric systems while keeping other unassigned poles unchanged. It uses state-feedback (active damping and active stiffness) to shift the poles to desired locations where all poles have negative real parts. Receptances at only a small, limited number of degrees-of-freedom of the underlying symmetric system are required. Simulated numerical examples indicate that this is an effective method and is capable of assigning negative real parts to unstable poles to stabilise an otherwise unstable second-order dynamic system.
\end{abstract}

Keywords: Partial pole assignment, asymmetric system, friction-induced vibration, flutter instability, receptance, state feedback

\section{Introduction}

Vibration in structures and machines is detrimental in many situations. Vibration may be reduced in a number of ways. One way is to shift the frequencies to some desirable values to avoid resonance. Another way is to assign zeros to certain locations of the system (structure or machine) for some excitation frequencies. Pole and zero assignment by means of structural modifications as a way of passive vibration control has been widely studied and applied, for example, in [1-4]. Active vibration control to assign poles and zeros has also attracted much attention [5-10]. Control engineers tend to use first-order equations. Although this is a general approach, it cannot take advantage of those peculiar properties afforded by the second-order equations, which are a natural formulation for vibration problems. It is believed that the second-order formulation for vibration problems can lead to new theories [11] and hence has been adopted by the authors.

Most researchers use mass, stiffness and sometimes damping matrices when assigning poles and zeros by active control [5-10], which is called a model-based approach. On the other hand, Ram and Mottershead [12] recently put forward a receptance-based inverse method for assigning poles and zeros to symmetric systems using state-feedback, which has distinct advantages over other methods for assigning poles and zeros. For example, the knowledge of mass, stiffness and damping matrices, though useful, is not required in this method, so that modelling errors can be avoided. This new idea was also applied to assign sensitivity of poles [13].

Conventional vibration problems are governed by second-order differential equations whose mass, damping and stiffness matrices are symmetric. Some structures and machines interact with internal nonconservative forces and make up asymmetric systems, whose stiffness and/or damping matrices are asymmetric. Friction-induced vibration,

\footnotetext{
*Corresponding author: H. Ouyang, School of Engineering, University of Liverpool, The Quadrangle, Liverpool L69 3GH, UK. E-mail: h.ouyang@liverpool.ac.uk.
} 
aeroelastic flutter and machining chatter belong to this category of dynamic systems. Asymmetric systems are prone to flutter instability as the real parts of some of their poles are positive as some parameters vary (for example, increase of flight speed for an aircraft). Through pole assignment for the unstable poles to gain negative real parts, an unstable asymmetric system can be stabilized.

Apart from [8], the afore-mentioned works all studied symmetric systems. In [8], Chu used a model-based approach. Ouyang [14] studied structural modifications to asymmetric systems for gaining stability based on receptances of the underlying symmetric systems. It was found that structural modifications as a major mean of passive vibration control is not very capable of assigning negative real parts of unstable poles to asymmetric systems. In [15,16], Ram and Mottershead's idea of [12] was extended to pole assignment of asymmetric systems by means of state feedback, again based on receptances. It was shown that active stiffness and active damping together, and active mass and active damping together, both are capable of assigning all poles (hence negative real parts) to asymmetric systems.

Real structures and machines always possess a large number of degrees of freedom. Consequently, full pole assignment is neither feasible nor necessary. This paper presents a receptance-based method for assigning a small number of significant poles of asymmetric system using state-feedback. It is a further extension to [12] and [15]. Datta et al. [6] considered partial pole assignment as a solution to the problem of spillover in structural control problems and this was developed for the receptance-based approach by Tehrani et al. [17]. In [6] those eigenvalues that remained unchanged from the open-loop condition were made unobservable whereas in [17] they became uncontrollable.

\section{Pole assignment by means of state feedback}

The Laplace transform of a second-order asymmetric system with an asymmetric stiffness matrix can be written as

$$
\left(\mathbf{M} s^{2}+\mathbf{C} s+\mathbf{K}+\sum_{i=1}^{j} \mu_{i} k_{c i} \mathbf{E}_{i}\right) \mathbf{x}(s)=\mathbf{p}(s)
$$

where $\mathbf{M}, \mathbf{C}$ and $\mathbf{K}$ are $n \times n$ symmetric mass, damping and stiffness matrices; $\mathbf{p}$ and $\mathbf{x}$ are respectively the Laplace transforms of the external load vector and displacement vector; for a friction-induced vibration problem, $\mu_{i}, k_{c i}$ and $\mathbf{E}_{i}$ are the friction coefficient at the $i$-th degree-of-freedom, and its associated stiffness term and location, respectively; and there are $j$ such asymmetric terms. Note that square matrix $\mathbf{E}_{i}$ has only one non-zero element, whose row corresponds to the tangential degree-of-freedom and whose column corresponds to the normal degree-of-freedom of the same node at the friction interface.

A single control force $u(\mathrm{~s})$ is introduced to influence the dynamic behaviour of equation as

$$
\left(\mathbf{M} s^{2}+\mathbf{C s}+\mathbf{K}+\sum_{i=1}^{j} \mu_{i} k_{c i} \mathbf{E}_{i}\right) \mathbf{x}(s)=\mathbf{p}(s)-\mathbf{b}(s) u(s)
$$

where $\mathbf{b}$ is the control force distribution vector.

Multiplying both sides of Eq. (2) by the transfer matrix of the symmetric part of the asymmetric system $\mathbf{H}(s)=\left(\mathbf{M} s^{2}+\mathbf{C} s+\mathbf{K}\right)^{-1}$ yields,

$$
\left(\mathbf{I}+\mathbf{H}(s) \sum_{i=1}^{j} \mu_{i} k_{c i} \mathbf{E}_{i}\right) \mathbf{x}(s)=\mathbf{H}(s)[\mathbf{p}(s)-\mathbf{b}(s) u(s)]
$$

Therefore the transfer matrix of the asymmetric system can be defined in terms of the transfer matrix of the underlying symmetric system and the friction coefficients and their associated stiffnesses as 


$$
\mathbf{H}_{a}(s)=\left(\mathbf{I}+\mathbf{H}(s) \sum_{i=1}^{j} \mu_{i} k_{c i} \mathbf{E}_{i}\right)^{-1} \mathbf{H}(s)
$$

and

$$
\mathbf{x}(s)=\mathbf{H}_{a}(s)(\mathbf{p}(s)-\mathbf{b}(s) u(s))
$$

It must be pointed out that it is important to use the transfer matrix of the underlying symmetric system $\mathbf{H}(s)$ because it is symmetric and much easier to measure in real applications. It would be more difficult to measure $\mathbf{H}_{a}(s)$ : for a disc brake, measuring $\mathbf{H}_{a}(s)$ requires applying a torque to try to turn the disc while the brake is engaged; for an aircraft wing, measuring $\mathbf{H}_{a}(s)$ requires testing it in a wind tunnel. Please also note that in reality the receptance matrix $\mathbf{H}(\mathrm{i} \omega$ ) (where $\mathrm{i}=\sqrt{-1}$ ) is measured, which readily gives $\mathbf{H}(s)$. So there is no need to distinguish the transfer matrix from receptance matrix in this paper.

State feedback is used to assign the poles of the asymmetric system in this investigation. The control force is considered to be in the form of velocity and displacement feedback as

$$
u(s)=(\mathbf{g}+s \mathbf{f})^{\mathrm{T}} \mathbf{x}(s)
$$

where $\mathbf{f}$ and $\mathbf{g}$ are called active damping and active stiffness vectors (gains). Other types of state feedback, for example, active damping and active mass, are equally applicable.

Now Eq. (5) becomes

$$
\left(\mathbf{I}+\mathbf{H}_{a}(s) \mathbf{b}(\mathbf{g}+s \mathbf{f})^{\mathrm{T}}\right) \mathbf{x}(s)=\mathbf{H}_{a}(s) \mathbf{p}(s)
$$

It follows

$$
\mathbf{x}(s)=\overline{\mathbf{H}}(s) \mathbf{p}(s)
$$

with the receptance matrix of the closed-loop system being $\overline{\mathbf{H}}(s)=\left(\mathbf{I}+\mathbf{H}_{a}(s)(\mathbf{g}+s \mathbf{f})\right)^{-1} \mathbf{H}_{a}(s)$. I is the identity matrix of appropriate dimension.

State feedback control in the form of Eq. (6) results in a rank-1 modification to the dynamic stiffness matrix. Using the Sherman-Morrison formula, $\overline{\mathbf{H}}(s)$ can be expressed in terms of the open-loop receptance matrix $\mathbf{H}_{a}(s)$ and the feedback control gains $\mathbf{f}$ and $\mathbf{g}$ as

$$
\overline{\mathbf{H}}(s)=\mathbf{H}_{a}(s)-\frac{\mathbf{H}_{a}(s) \mathbf{b}(\mathbf{g}+s \mathbf{f})^{\mathrm{T}} \mathbf{H}_{a}(s)}{1+(\mathbf{g}+s \mathbf{f})^{\mathrm{T}} \mathbf{H}_{a}(s) \mathbf{b}}
$$

The poles of the closed-loop system causes the denominator in Eq. (9) to vanish, that is,

$$
1+(\mathbf{g}+s \mathbf{f})^{\mathrm{T}} \mathbf{H}_{a}(s) \mathbf{b}=0
$$

For full pole assignment, the $2 n$ desired poles are substituted into Eq. (9) and $2 n$ equations in the $n$ elements of $\mathbf{f}$ and $n$ elements of $\mathbf{g}$ can be derived to fully determine the required $\mathbf{f}$ and $\mathbf{g}$ [15] after $\mathbf{b}$ is specified.

\section{Partial pole placement using the controllability condition}

Full pole assignment means solving $2 \times n$ equations to assign all the poles, which is expensive and unnecessary, because only a few poles would need to be reassigned in a real application. In partial pole placement, some poles are assigned to desirable values (note that negative real parts are more important for asymmetric systems) while the rest remains unchanged. This is obviously a more desirable solution. 
From Eq. (9), it can be seen that if a particular pole $\lambda_{k}$ makes the numerator in the second block of terms on the right-hand side vanish, then $\overline{\mathbf{H}}\left(\lambda_{k}\right)=\mathbf{H}_{a}\left(\lambda_{k}\right)$. This means that this particular pole remains unchanged even though the system is subjected to a certain control. The numerator can vanish when either

$$
\mathbf{H}_{a}\left(\lambda_{k}\right) \mathbf{b}=\mathbf{0}
$$

or

$$
\left(\mathbf{g}+\lambda_{k} \mathbf{f}\right)^{\mathrm{T}} \mathbf{H}_{a}\left(\lambda_{k}\right)=\mathbf{0}
$$

These two equations concern controllability and observability respectively. When either of these two conditions is satisfied by certain closed-loop poles then these poles become equal to the open-loop poles and hence remain unchanged. Equation (11) is used in this paper to 'constrain' these poles. In summary, the $l$ number of poles to be assigned to new desirable values $\lambda_{k}(k=1,2, \ldots, l)$ must satisfy Eq. (10) while the rest of poles $\lambda_{k}(k=l+1, l+2, \ldots, n)$ must satisfy Eq. (11).

Vector $\mathbf{b}$ is complex in general. It is decomposed into the following form in consideration of hardware implementation of the control force,

$$
\mathbf{b}\left(\lambda_{k}\right)=\mathbf{b}_{1}+\mathbf{b}_{2} \lambda_{k}
$$

where $\mathbf{b}_{1}$ and $\mathbf{b}_{2}$ are real vectors to be determined from Eq. (11). This means

$$
\left[\begin{array}{ll}
\mathbf{H}_{a}\left(\lambda_{k}\right) & \lambda_{k} \mathbf{H}_{a}\left(\lambda_{k}\right)
\end{array}\right]\left(\begin{array}{l}
\mathbf{b}_{1} \\
\mathbf{b}_{2}
\end{array}\right)=\mathbf{0}
$$

or

$$
\left(\begin{array}{c}
\mathbf{b}_{1} \\
\mathbf{b}_{2}
\end{array}\right)=\mathbf{V} \boldsymbol{\alpha} ; \quad \boldsymbol{V}=\operatorname{null}\left[\mathbf{H}_{a}\left(\lambda_{k}\right) \quad \lambda_{k} \mathbf{H}_{a}\left(\lambda_{k}\right)\right]=\operatorname{null}\left[\begin{array}{ll}
\mathfrak{R}\left(\mathbf{H}_{a}\left(\lambda_{k}\right)\right) & \mathfrak{R}\left(\lambda_{k} \mathbf{H}_{a}\left(\lambda_{k}\right)\right) \\
\mathfrak{I}\left(\mathbf{H}_{a}\left(\lambda_{k}\right)\right) & \mathfrak{I}\left(\lambda_{k} \mathbf{H}_{a}\left(\lambda_{k}\right)\right)
\end{array}\right]
$$

in which the terms in vector $\boldsymbol{\alpha}$ are used to select the columns of matrix $\mathbf{V}$ so that the complex conjugate pairs of poles $\left\{\lambda_{k}, \lambda_{k}^{*}\right\}(k=l+1, l+2, n)$ are uncontrollable (remain unchanged). 'Null' means the null space, and $\Re$ and $\mathfrak{I}$ represent real and imaginary parts respectively.

For those pairs of poles to be assigned new values $\left\{\lambda_{k}, \lambda_{k}^{*}\right\}(k=1,2, \ldots, l)$, it follows from Eq. (10) that

$$
1+\left(\mathbf{g}+\lambda_{k} \mathbf{f}\right)^{\mathrm{T}} \mathbf{H}_{a}\left(\lambda_{k}\right) \mathbf{b}=0, \quad 1+\left(\mathbf{g}+\lambda_{k}^{*} \mathbf{f}\right)^{\mathrm{T}} \mathbf{H}_{a}\left(\lambda_{k}^{*}\right) \mathbf{b}=0 \quad(k=1,2, \ldots, l)
$$

leading to

$$
\left[\begin{array}{cc}
\mathbf{b}^{\mathrm{T}} \mathbf{H}_{\mathrm{a}}^{\mathrm{T}}\left(\lambda_{1}\right) & \lambda_{1} \mathbf{b}^{\mathrm{T}} \mathbf{H}_{\mathrm{a}}^{\mathrm{T}}\left(\lambda_{1}\right) \\
\mathbf{b}^{\mathrm{T}} \mathbf{H}_{\mathrm{a}}^{\mathrm{T}}\left(\lambda_{1}^{*}\right) & \lambda_{1}^{*} \mathbf{b}^{\mathrm{T}} \mathbf{H}_{\mathrm{a}}^{\mathrm{T}}\left(\lambda_{1}^{*}\right) \\
\cdot & \cdot \\
\cdot & \cdot \\
\cdot & \cdot \\
\cdot & \cdot \\
\mathbf{b}^{\mathrm{T}} \mathbf{H}_{\mathrm{a}}^{\mathrm{T}}\left(\lambda_{1}\right) & \lambda_{1} \mathbf{b}^{\mathrm{T}} \mathbf{H}_{\mathrm{a}}^{\mathrm{T}}\left(\lambda_{1}\right) \\
\mathbf{b}^{\mathrm{T}} \mathbf{H}_{\mathrm{a}}^{\mathrm{T}}\left(\lambda_{1}^{*}\right) & \lambda_{1}^{*} \mathbf{b}^{\mathrm{T}} \mathbf{H}_{\mathrm{a}}^{\mathrm{T}}\left(\lambda_{1}^{*}\right)
\end{array}\right]\left\{\begin{array}{c}
\mathbf{g} \\
\mathbf{f}
\end{array}\right\}=\left\{\begin{array}{c}
-1 \\
-1 \\
\cdot \\
\cdot \\
\cdot \\
\cdot \\
-1
\end{array}\right\}
$$


Equation (17) is an under-determined equation that allows an infinite number of solutions of elements of $\mathbf{f}$ and $\mathbf{g}$. The pseudo-inverse is used to obtain a least-squares solution.

In this way the poles of the closed-loop system are separated into assigned poles and unassigned poles to achieve partial pole placement. It can be seen from the above equations that there is no need to know or to evaluate the system matrices $\mathbf{M}, \mathbf{C}$ and $\mathbf{K}$, which is consistent with the approach described by Ram and Mottershead [12], based on measured receptances from vibration tests.

\section{Simulate numerical example}

A mass-spring structure standing on a rigid belt moving at constant speed is shown in Fig. 1. The system has three masses with $m_{1}$ having a degree-of-freedom in the $x$ (horizontal) direction, $x_{1} ; m_{3}$ having a degree-of-freedom in the $y$ (vertical) direction, $y_{2}$; and $m_{2}$ having degrees-of-freedom in both directions, $x_{2}$ and $y_{2} . f_{1}$ and $n_{1}$ are respectively the friction force and (pre-compression) normal force acting at the slider-belt interface. It is assumed that there is a high enough pre-compression normal force there to maintain contact during vibration. The sliding friction at the slider-belt interface is governed by Coulomb friction whose static and kinetic friction coefficients are taken to be the same. This is a simplification and avoids non-smooth stick-slip vibration. This example was used in full pole assignment in [15].

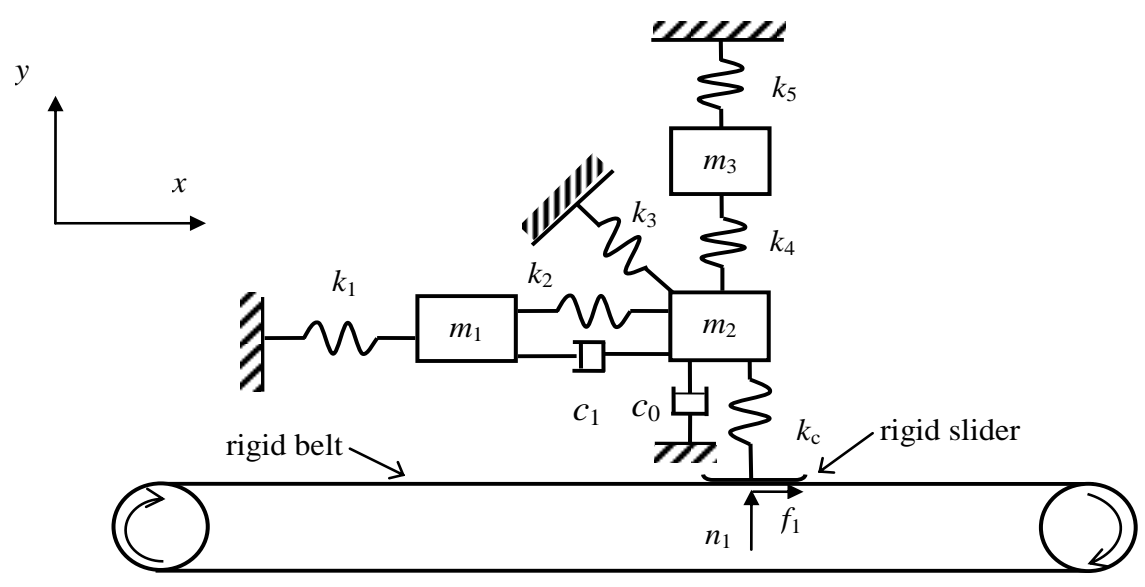

Fig. 1. A 4-degree-of-freedom friction-induced vibration problem.

For displacement vector $\mathbf{x}^{\mathrm{T}}=\left\{\begin{array}{llll}x_{1} & y_{3} & x_{2} & y_{2}\end{array}\right\}$, the mass, damping and stiffness matrices are:

$$
\begin{aligned}
\mathbf{M} & =\left[\begin{array}{ccccc}
m_{1} & & & \\
& m_{3} & & \\
& & m_{2} & \\
& & m_{2}
\end{array}\right] \mathbf{C}=\left[\begin{array}{cccc}
c_{1} & 0 & -c_{1} & 0 \\
0 & 0 & 0 & 0 \\
-c_{1} & 0 & c_{1} & 0 \\
0 & 0 & 0 & c_{0}
\end{array}\right] \\
\mathbf{K} & =\left[\begin{array}{ccccc}
k_{1}+k_{2} & 0 & -k_{2} & 0 & \\
0 & k_{4}+k_{5} & 0 & 0 & \\
-k_{2} & 0 & k_{2}+0.5 k_{3} & 0.5 k_{3} \\
0 & 0 & 0.5 k_{3} & k_{4}+0.5 k_{3}+k_{c}
\end{array}\right] \mathbf{E}=\left[\begin{array}{cccc}
0 & 0 & 0 & 0 \\
0 & 0 & 0 & 0 \\
0 & 0 & 0 & 1 \\
0 & 0 & 0 & 0
\end{array}\right]
\end{aligned}
$$


where $m_{i}=1 \mathrm{~kg}(i=1,2,3), c_{i}=0.5 \mathrm{Ns} / \mathrm{m}(i=0,1) k_{i}=100 \mathrm{~N} / \mathrm{m}(i=1,2,3,4,5)$ and $k_{c}=2 k_{1}$.

The open-loop poles for the asymmetric system with the friction coefficient of $\mu=0.5$ and the assigned poles are listed in Table 1 below.

Table 1

Open-loop poles and the desired closed poles at $\mu=0.5$

\begin{tabular}{ccccc}
\hline \multicolumn{1}{c}{ Poles } & $\lambda_{1,2}$ & $\lambda_{3,4}$ & $\lambda_{5,6}$ & $\lambda_{7,8}$ \\
\hline Open-loop & $0.007 \pm 8.9462 \mathrm{i}$ & $-0.0553 \pm 12.1336 \mathrm{i}$ & $-0.5259 \pm 16.8229 \mathrm{i}$ & $-0.1757 \pm 19.7336 \mathrm{i}$ \\
Closed-loop & $-0.0800 \pm 10.0000 \mathrm{i}$ & $-0.1000 \pm 14.0000 \mathrm{i}$ & $-0.5259 \pm 16.8229 \mathrm{i}$ & $-0.1757 \pm 19.7336 \mathrm{i}$ \\
\hline
\end{tabular}

Notice that in Table 1, the first two pairs of poles are assigned to new values and the rest remains unchanged. Vectors $\mathbf{b}_{1}$ and $\mathbf{b}_{2}$ are obtained from the null-space of the unassigned open-loop eigenvectors in

$$
\left[\begin{array}{ll}
\mathbf{H}_{a}\left(\lambda_{5}\right) & \lambda_{5} \mathbf{H}_{a}\left(\lambda_{5}\right) \\
\mathbf{H}_{a}\left(\lambda_{7}\right) & \lambda_{7} \mathbf{H}_{a}\left(\lambda_{7}\right)
\end{array}\right]\left(\begin{array}{l}
\mathbf{b}_{1} \\
\mathbf{b}_{2}
\end{array}\right)=\mathbf{0}
$$

where

$$
\operatorname{null}\left(\begin{array}{ll}
\mathfrak{R}\left(\begin{array}{ll}
\mathbf{H}_{a}\left(\lambda_{5}\right) & \lambda_{5} \mathbf{H}_{a}\left(\lambda_{5}\right) \\
\mathbf{H}_{a}\left(\lambda_{7}\right) & \lambda_{7} \mathbf{H}_{a}\left(\lambda_{7}\right)
\end{array}\right) \\
\mathfrak{I}\left(\begin{array}{ll}
\mathbf{H}_{a}\left(\lambda_{5}\right) & \lambda_{5} \mathbf{H}_{a}\left(\lambda_{5}\right) \\
\mathbf{H}_{a}\left(\lambda_{7}\right) & \lambda_{7} \mathbf{H}_{a}\left(\lambda_{7}\right)
\end{array}\right)
\end{array}\right)=\left[\begin{array}{cccc}
-0.1359 & -0.1385 & 0.0465 & -0.6198 \\
0.1468 & -0.0052 & 0.8927 & -0.0059 \\
0.1427 & 0.0998 & -0.1122 & -0.7752 \\
-0.2499 & 0.0486 & 0.4340 & -0.1219 \\
-0.0779 & 0.6083 & 0.0002 & -0.0018 \\
-0.8348 & -0.0265 & 0.0006 & 0.0032 \\
0.0633 & 0.7659 & 0.0015 & -0.0020 \\
-0.4126 & 0.1052 & -0.0004 & 0.0014
\end{array}\right]
$$

The following $\mathbf{b}$ can be obtained

$$
\mathbf{b}=\left(\begin{array}{c}
-0.1359 \\
0.1468 \\
0.1427 \\
-0.2499
\end{array}\right)+s\left(\begin{array}{c}
-0.0779 \\
-0.8348 \\
0.0633 \\
-0.4126
\end{array}\right)
$$

When the above $\mathbf{b}$ and the desired first two pairs of poles are substituted into Eq. (16), the required control gains are found to be,

$$
\mathbf{g}=\left(\begin{array}{c}
0.7939 \\
-0.1655 \\
1.0224 \\
0.0771
\end{array}\right), \mathbf{f}=\left(\begin{array}{c}
-0.7469 \\
0.4484 \\
-0.8670 \\
0.0665
\end{array}\right)
$$


When $\mathbf{b}, \mathbf{g}$ and $\mathbf{f}$ from the above are substituted into Eq. (9), the receptance matrix of the closed-loop control system is obtained. Figure 2 shows the open-loop and closed-loop receptance terms $h_{a 11}$ and $\bar{h}_{11}$, and $h_{a 33}$ and $\bar{h}_{33}$ of the asymmetric system of Fig. 1, where it can be seen that the first two peaks correspond to the frequencies of the first two pairs of assigned poles and the other two peaks to the frequencies of the other two pairs of retained poles.

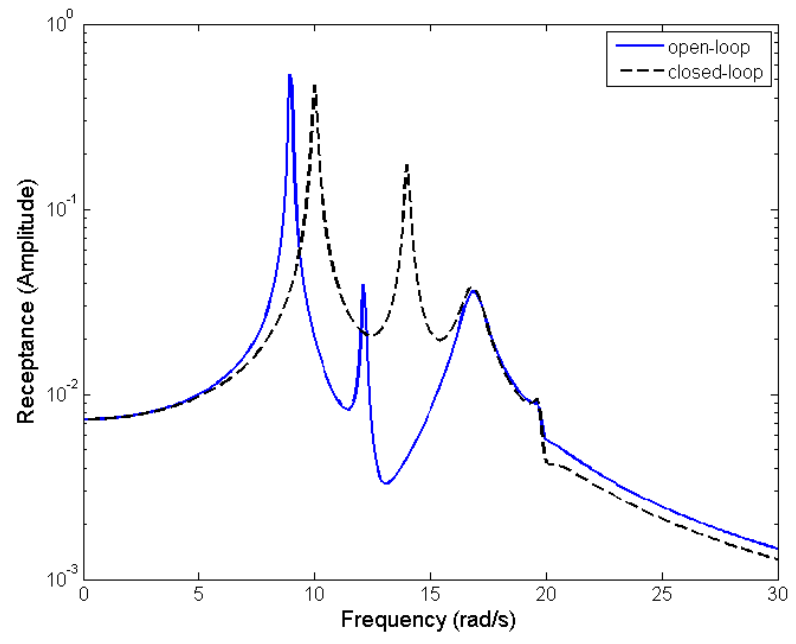

(a)

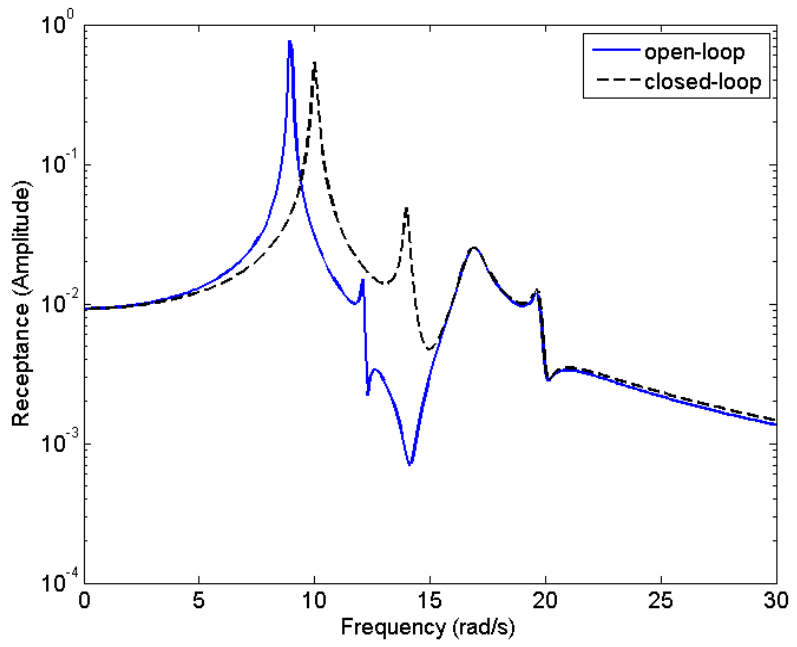

(b)

Fig. 2. Open-loop and closed-loop receptances: (a) $h_{a 11}$ and $\bar{h}_{11}$ and (b) $h_{a 33}$ and $\bar{h}_{33}$.

It should be pointed out that if instead dashpot $c_{0}$ is connected to the slider in parallel with $k_{c}$, then the resultant system would have an asymmetric stiffness matrix and an asymmetric damping matrix, and the method presented above is equally applicable. The method is also applicable to active control using multiple control forces.

\section{Conclusions}

This paper presents an inverse receptance-based method for determining the control gains required for partial pole assignment of asymmetric systems by means of state feedback control. A four-degrees-of-freedom, friction-induced vibration problem is used as an example to demonstrate the application of this method and its effectiveness. Because of the use of receptances, which can easily be measured, there is no modelling error involved. An unstable asymmetric dynamic system can be stabilised by assigning those unstable poles with negative real parts while keeping the stable poles unchanged.

\section{References}

[1] I. Bucher and S. Braun, The structural modification inverse problem: an exact solution, Mechanical Systems and Signal Processing 7(3) (1993), 217-238.

[2] A. Kyprianou, J.E. Mottershead and H. Ouyang, Structural modification, part 2: Assignment of natural frequencies and antiresonances by an added beam, Journal of Sound and Vibration 284(1,2) (2005), 267-281.

[3] M.G. Tehrani, W. Wang, C. Mares and J.E. Mottershead, The generalized Vincent circle in vibration suppression, Journal of Sound and Vibration 292(3-5) (2006), 661-675.

[4] J.E. Mottershead, M.G. Tehrani, D. Stancioiu, S. James and H. Shahverdi, Structural modification of a helicopter tailcone, Journal of Sound and Vibration 298(1,2) (2006), 366-384.

[5] E.K. Chu and B.N. Datta, Numerically robust pole assignment for second order systems, International Journal of Control 64(4) (1996), 1113-1127.

[6] B.N. Datta, S. Elhay, Y.M. Ram and D.R. Sarkissian, Partial eigenstructure assignment for the quadratic pencil, Journal of Sound and 
Vibration 230(1) (2000), 101-110.

[7] V.K. Singh and Y. Ram, Dynamic absorption by passive and active control, ASME Journal of Vibration and Acoustics 122(5) (2000), 429-433.

[8] E.K. Chu, Pole assignment for second-order systems, Mechanical Systems and Signal Processing 16(1) (2002), 39-59.

[9] G.R. Duan and Y.L. Wu, Robust pole assignment in matrix descriptor second-order linear systems, Transactions of the Institute of measurement and Control 27(4) (2005), 279-295.

[10] Y.M. Ram, A. Singh and J.E. Mottershead, State feedback control with time delay, Mechanical Systems and Signal Processing 23 (2009), 1940-1945.

[11] D.J. Inman, Active modal control for smart structures, Philosophical Transactions of the Royal Society of London A 359(1778) (2001), 205-219.

[12] Y.M. Ram and J.E. Mottershead, Receptance method in active vibration control, American Institute of Aeronautics and Astronautics Journal 45(3) (2007), 562-567.

[13] J.E. Mottershead, M.G. Tehrani and Y.M. Ram, Assignment of eigenvalue sensitivities from receptance measurements, Mechanical Systems and Signal Processing 23(6) (2009), 1931-1939.

[14] H. Ouyang, Prediction and assignment of latent roots of damped asymmetric systems by structural modifications, Mechanical Systems and Signal Processing 23(6) (2009), 1920-1930.

[15] H. Ouyang, Pole assignment of friction-induced vibration for stabilisation through state-feedback control, Journal of Sound and Vibration 329(11) (2010), 1985-1991.

[16] H. Ouyang, A hybrid control approach for pole assignment to second-order asymmetric systems, Mechanical Systems and Signal Processing 25(1) (2011), 123-132.

[17] M.G. Tehrani, R.N.R. Elliott and J.E. Mottershead, Partial pole placement in structures by the method of receptances: Theory and experiments, Journal of Sound and Vibration 329(24) (2010), 5017-5035. 

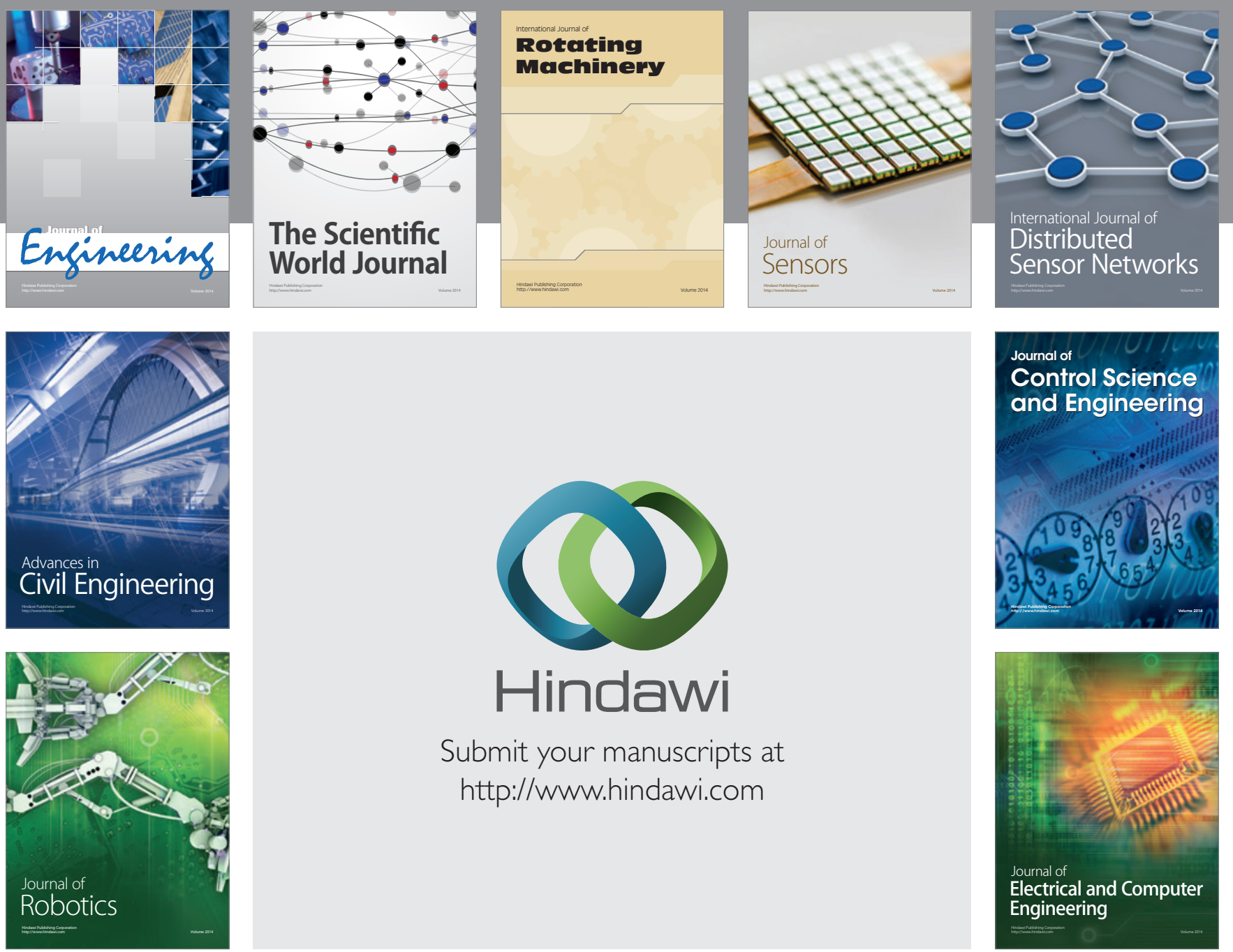

Submit your manuscripts at

http://www.hindawi.com
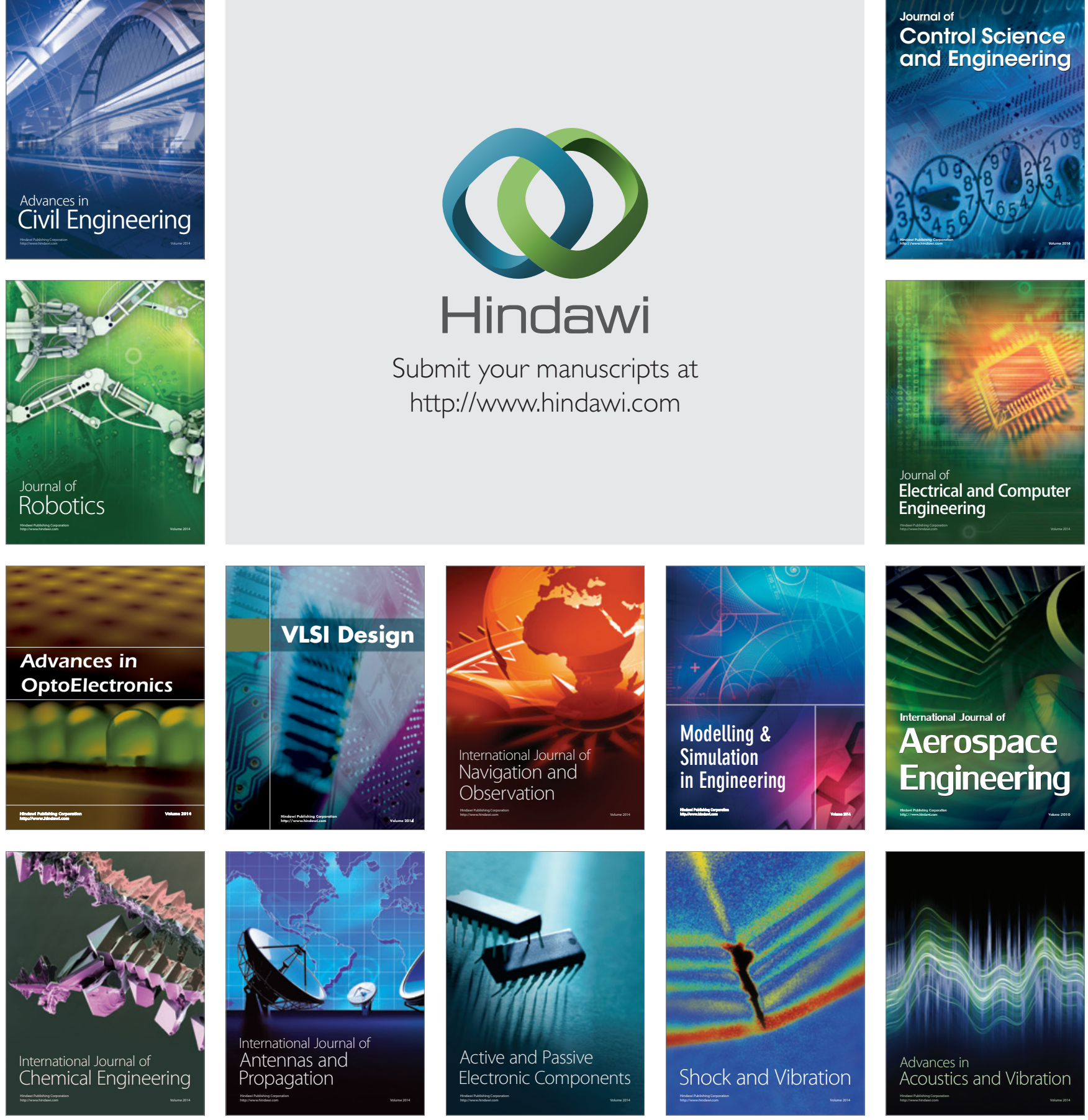